\title{
Decrease in Multidrug Resistance-associated Protein 2 Activities by Knockdown of Phosphatidylinositol 4-phosphate 5-kinase in Hepatocytes and Cancer Cells
}

\author{
Atsushi Kawase ${ }^{1}$, Yuta Inoue ${ }^{1}$, Miho Hirosoko ${ }^{1}$, Yuka Sugihara ${ }^{1}$, Hiroaki Shimada ${ }^{1}$, Masahiro Iwaki $^{1,2,3}$ \\ 1. Department of Pharmacy, Faculty of Pharmacy, Kindai University, Osaka, Japan. ${ }^{2}$ Pharmaceutical Research and \\ Technology Institute, Kindai University, Osaka, Japan. ${ }^{3 \cdot}$ Antiaging Center, Kindai University, Osaka, Japan.
}

Receive, July 21, 2019; Revised, October 17, Accepted, October 19; Published, October 20, 2019.

\begin{abstract}
Purpose: The plasma membrane localization and transport activity of multidrug resistanceassociated protein 2 (MRP2/ABCC2) and P-glycoprotein (P-gp/ABCB1) efflux transporters are governed by transporter-associated proteins. Phosphatidylinositol 4,5-bisphosphate $\left(\mathrm{PIP}_{2}\right)$ formed by phosphatidylinositol 4-phosphate 5-kinase type 1 (PIP5K1) activates the linker function of radixin for efflux transporters. Radixin is involved in the plasma membrane localization of efflux transporters. We examined whether PIP5K1 could be a target for the modulation of transporter activities in hepatocytes and cancer cells. Methods: The effects of PIP5K1 depletion by siRNA in mouse primary hepatocytes, PANC1 human pancreatic carcinoma cells, and HepG2 human hepatocellular carcinoma cells on the intracellular accumulation of MRP2 and P-gp substrates were examined. Results: PIP5K1A depletion resulted in increased intracellular accumulation of carboxydichlorofluorescein, a MRP2 fluorescent substrate, in mouse primary hepatocytes, PANC1 cells, and HepG2 cells. In PANC1 and HepG2 cells, the transport activities of MRP2 were significantly decreased by PIP5K1C depletion. However, the transport activities of P-gp were unchanged by PIP5K1 depletion. PIP 2 levels were unchanged between control and PIP5K1A- or PIP5K1C-depleted HepG2 cells. MRP2 mRNA levels showed few changes in HepG2 cells following PIP5K1A or PIP5K1C depletion. The expression of phosphorylated radixin was decreased by PIP5K1A and PIP5K1C depletion, although total radixin levels were unchanged. Conclusions: These data suggest that PIP5K1A and PIP5K1C could be target proteins for modulating MRP2 function, partly because of the resulting changes of the linker function of radixin.
\end{abstract}

\section{PURPOSE}

ATP-binding cassette $(\mathrm{ABC})$ transporters such as multidrug resistance-associated protein 2 (MRP2/ABCC2) and P-glycoprotein (P-gp/ABCB1), which function as efflux transporters, are determinants of the pharmacokinetics of some drugs(1-3). In the blood-brain barrier, P-gp and MRP2 transport intracellular drugs to the blood to protect the central nervous system(4). In the liver, MRP2 expressed on the canalicular membrane participates in the biliary excretion of bile acid and drug metabolites, and MRP2 dysfunction due to genetic diseases or drug inhibition causes intrahepatic cholestasis(5). The overexpression of MRPs and P-gp in cancer cells causes resistance to anti-cancer drugs such as paclitaxel, etoposide, and doxorubicin $(1-3,6)$.

The plasma membrane localization and transport activity of efflux transporters is governed by transporter-associated proteins such as phosphatidylinositol 4,5-bisphosphate ( $\left.\mathrm{PIP}_{2}\right)$, the ezrin/radixin/moesin (ERM) family proteins, and $\mathrm{Na}^{+} / \mathrm{H}^{+}$exchanger regulating factor $1 /$ ERM binding phosphoprotein of $50 \mathrm{kDa}(7-9)$. ERM proteins are activated by C-terminal phosphorylation by $\mathrm{PIP}_{2}$ and subsequently function as a linker between efflux transporters and actin filaments(8,10-13). Although the involvement of ERMs such as radixin in the plasma membrane localization and transport activity of MRP2 is well defined(14), few reports have examined the relation between $\mathrm{PIP}_{2}$ activity and MRP2.

The membrane phospholipid $\mathrm{PIP}_{2}$ is involved in a variety of cellular signaling pathways, such as cytoskeletal signaling, tissue remodeling, and vesicle trafficking(15), and regulates the membrane localization of ion channels and transporters $(16,17)$.

Corresponding Author: Atsushi Kawase, Ph.D., Department of Pharmacy, Faculty of Pharmacy, Kindai University, 3-4-1 Kowakae, Higashi-osaka, Osaka 577-8501, Japan. E-mail: kawase@phar.kindai.ac.jp 
$\mathrm{PIP}_{2}$ is produced by the phosphorylation of phosphatidylinositol 4-phosphate (PI(4)P) or phosphatidylinositol 5-phosphate $(\mathrm{PI}(5) \mathrm{P})$ and dephosphorylation of phosphatidylinositol $(3,4,5)$ trisphosphate(18-21). Phosphorylation of PI(4)P by the lipid kinase phosphatidylinositol 4-phosphate 5kinase type 1 (PIP5K1) is the predominant pathway for $\mathrm{PIP}_{2}$ biosynthesis(22). The intracellular levels of PI(4)P are approximately 10-fold higher than those of PI(5)P. PIP5K1 has three isozymes: PIP5K1A (human PIP5K $\alpha$ and mouse PIP5K $\beta$ ), PIP5K1B (human PIP5K $\beta$ and mouse PIP5K $\alpha$ ), and PIP5K1C (human and mouse PIP5K $\gamma$ ). Previous studies showed that PIP5K1A overexpression is involved in the malignant transformation of prostate cancer cells(23). Cytosolic PIP5K1A mainly participates in the generation of $\mathrm{PIP}_{2}$ in humans(24). PIP5K1Bknockout mice exhibited anaphylactic responses caused by increased cytokine production by mast cells(25). PIP5K1C is highly expressed in the brain and is involved in the endocytosis-mediated recovery of synaptic vesicles(26). PIP5K1C binds to the scaffold protein talin to promote integrin expression on the plasma membrane(27). Previous studies showed that the activities of PIP5Ks are higher in cancer cells than in normal cells(28). However, the roles of PIP5K1A, PIP5K1B, and PIP5K1C in the transport activity of MRP2 remain unclear.

Determining the potential function of PIP5Ks in regulating MRP2 function may help elucidate MRP2 interacting proteins and other related pathways in hepatocytes and cancer cells. Therefore, here we investigated whether PIP5K1A, PIP5K1B, or PIP5K1C are involved in the transport activity of MRP2 in hepatocytes and cancer cells.

\section{METHODS}

\section{Chemicals and reagents}

Dulbecco's modified Eagle's medium (DMEM), RPMI-1640, Sepasol RNA I Super G, and Hanks' balanced salt solution were purchased from Nacalai Tesque (Kyoto, Japan). Silencer Select siRNA for mouse PIP5K1A (s71636), human PIP5K1A (s15932), human PIP5K1B (s15934), and human PIP5K1C (s23732); siRNA negative control Med GC (siNegative); Opti-MEM medium; Lipofectamine RNAiMAX; GlutaMAX supplement I; Fast SYBR Green Master Mix; Mem-PER eukaryotic membrane protein extraction reagent kit; and $\mathrm{BCA}$ protein assay kit were purchased from Thermo Fisher Scientific (Waltham, MA, USA). We used 5 (and 6)-carboxy-2',7'-dichlorofluorescein diacetate (CDFDA, an MRP substrate, SigmaAldrich, St. Louis, MO, USA), rhodamine 123 (R123, a P-gp substrate, Wako Pure Chemical Industries, Osaka, Japan), MK571 sodium salt hydrate (an MRP inhibitor, Sigma-Aldrich), and verapamil (a P-gp inhibitor, Nacalai Tesque) to assess transport activities. ReverTra Ace qPCR RT Master Mix was purchased from Toyobo (Osaka, Japan). Primers for target genes were purchased from Eurofin Genomics (Tokyo, Japan). RIPA lysis buffer including protease inhibitors was obtained from Santa Cruz Biotechnology (Dallas, TX, USA). Hybond-P polyvinylidene difluoride membranes, ECL Prime blocking reagent, and ECL Prime western blotting detection reagent were purchased from GE Healthcare (Milwaukee, WI, USA). Mouse monoclonal anti-PIP ${ }_{2}$ antibody (2C11) (Abcam, Cambridge, UK), rabbit monoclonal anti-radixin (Abcam), mouse monoclonal anti- $\square$-actin (Abcam), anti-mouse IgG antibody (peroxidase-labeled goat anti-mouse IgG, KPL, Gaithersburg, MD, USA), and anti-rabbit IgG (KPL, Gaithersburg, MD, USA) were commercially obtained. Phos-tag was obtained from NARD Institute (Hyogo, Japan). All other chemicals and solvents were of the highest commercially available purity.

\section{Cell culture}

Hepatocytes were isolated via two-step collagenase perfusion as described previously(29). Hepatocytes were seeded at $1 \times 10^{5}$ cells $/ \mathrm{cm}^{2}$ in collagen-coated plates (Sumitomo Bakelite, Tokyo, Japan) in Williams' Medium E supplemented with $10 \%$ fetal bovine serum, $1 \mathrm{nM}$ insulin, $1 \mathrm{nM}$ dexamethasone, $100 \mathrm{U} / \mathrm{ml}$ penicillin, $100 \mu \mathrm{g} / \mathrm{ml}$ streptomycin, and 2 mM GlutaMAX. Cells were incubated at $37^{\circ} \mathrm{C}$ in the presence of $5 \% \mathrm{CO}_{2}$ and $95 \%$ air. After cell attachment for $3 \mathrm{~h}$, the medium was replaced with fresh serum-free Williams' Medium E containing the aforementioned supplements and $5 \mathrm{U} / \mathrm{ml}$ aprotinin.

Human pancreas cancer PANC1 and human liver cancer HepG2 cells (obtained from RIKEN Cell Bank, Ibaraki, Japan) were maintained in RPMI-1640 and DMEM, respectively, supplemented with $10 \%$ fetal bovine serum, 100 $\mathrm{U} / \mathrm{ml}$ penicillin, and $100 \mu \mathrm{g} / \mathrm{ml}$ streptomycin. Cells were grown at $37^{\circ} \mathrm{C}$ in a humidified incubator equilibrated with $5 \% \mathrm{CO}_{2}$ and were subcultured every 3-4 days using $0.25 \%$ trypsin and $1 \mathrm{mM}$ EDTA. The medium was replaced every 2 days. PANC1 and HepG2 cells were seeded at $2.5 \times 10^{4}$ cells $/ \mathrm{cm}^{2}$ in 24-well plates. 
Transfection of siRNA

Mouse primary hepatocytes were treated with 5 pmol/well siRNA targeting PIP5K1A (siPIP5K1A) or siNegative complexed with Lipofectamine RNAiMAX in Opti-MEM. PANC1 cells were transfected with siRNA (siPIP5K1A, siPIP5K1B, siPIP5K1C, siNegative; $5 \mathrm{pmol} /$ well) complexed with Lipofectamine RNAiMAX in Opti-MEM. In HepG2 cells, reverse transfection (the addition of cells to siRNA complexes [5 pmol/well] on a plate) was performed. At $48 \mathrm{~h}$ after transfection, the cells were used for experiments.

Real-time reverse transcription polymerase chain reaction (RT-PCR)

Total RNA was extracted from mouse primary hepatocytes, PANC1 cells and HepG2 cells using Sepasol RNA I Super G. The mRNA expression was measured using RT-PCR, as described previously(30,31). The oligonucleotide sequences for each mRNA target were as follows: mouse PIP5K1A (forward: 5'CTTGCCTCGGTCAGTCAAAA-3', reverse: 5'AGCATCCAAAAATAGGCCGT-3'); human PIP5K1A (forward: 5'GAACACAAGGCACAAGTGACAAC-3', reverse: 5'-AAAACATCAGGACGACCAAGG-3'); human PIP5K1B (forward: 5'GACTGCCGGGTGCTAGAAAG-3', reverse: 5'GGGAATGGTCCAGGAAATGA-3'); human PIP5KC (forward: 5'CTGTCGCCTTCCGCTACTTC-3', reverse: 5'GGCTCATTGCACAGGGAGTAC-3'); human MRP2 (forward: 5'-ATGCTTCCTGGGGATAAT3', reverse: 5'-TCAAAGGCACGGATAACT-3'); human P-gp (forward: 5'CTGTATTGTTTGCCACCACGA-3', reverse: 5'AGGGTGTCAAATTTATGAGGCAGT-3');

mouse $\quad \beta$-actin $\quad$ (forward: 5 'CCGATCCACACAGAGTACTTGC-3', reverse: 5'CATTGCTGCAGGATGCAGAA-3'), human GAPDH (forward: 5'AGGGCTGCTTTTAACTCTGGT-3', reverse: 5'CCCCACTTGATTTTGGAGGG-3'). Data were analyzed using the StepOne Real-Time PCR System (Thermo Fisher Scientific) using the multiplex comparative method. Target mRNA levels were normalized to $\beta$-actin mRNA and GAPDH mRNA for mouse and human genes, respectively.

Determination of expression of PIP5K1A, PIP5K1B and PIP5K1C by targeted proteomics The protein expression levels of PIP5K1A, PIP5K1B and PIP5K1C were determined by targeted proteomics as described previously $(32,33)$. Briefly, cell lysates $(50 \square \mathrm{g})$ diluted in $25 \square 1$ phase transfer surfactant buffer were treated with $10 \mathrm{mM}$ dithiothreitol and $42 \mathrm{mM}$ iodoacetamide and digested by $1 \square \mathrm{g}$ trypsin at $37^{\circ} \mathrm{C}$ for $18 \mathrm{~h}$. Peptides were separated on a reversed-phase column (AdvanceBio Peptide Map C18, $2.1 \times 150$ mm, 2.7 $\square \mathrm{m}$; Agilent Technologies, Richardson, TX, USA) and detected on a LC-MS/MS apparatus consisting of an LC system (UltiMate 3000 series, Thermo Fisher Scientific, Waltham, MA, USA) and a TSQ Endura Triple Quadrupole Mass Spectrometer with electrospray ionization (Thermo Fisher Scientific).

\section{Functional studies of MRP2 and P-gp with fluorescent substrate}

Hepatocytes, HepG2 cells, and PANC1 cells were treated with siPIP5K1A, siPIP5K1B, siPIP5K1C, or siNegative for $48 \mathrm{~h}$; the medium was removed and the cells were incubated in Hanks' balanced salt solution containing MK571 $(50 \mu \mathrm{M})$ and verapamil $(50 \square \mathrm{M})$ for 30 or $60 \mathrm{~min}$ at $37^{\circ} \mathrm{C}$. CDFDA $(10 \mu \mathrm{M})$ and R123 (50 $\square \mathrm{M})$ was added to the cells, and cells were incubated for 60 or $120 \mathrm{~min}$ at $37^{\circ} \mathrm{C}$. The medium was removed, and cells were washed twice with ice-cold phosphate-buffered saline. Next, 100 $\mu l$ of $1 \mathrm{M} \mathrm{NaOH}$ were added, and the cell lysate was collected using a cell scraper. The fluorescence intensity $(\mathrm{Ex} / \mathrm{Em}=485 \mathrm{~nm} / 530 \mathrm{~nm})$ derived from carboxydichlorofluorescein (CDF) or R123 was measured using a SH-9000Lab fluorescent microplate reader (Corona Electric Co., Ibaraki, Japan).

\section{Preparation of cell lysates and membrane proteins}

Cell lysates were isolated from HepG2 cells at $48 \mathrm{~h}$ after siRNA transfection using RIPA lysis buffer. Membrane proteins were extracted using the MemPER eukaryotic membrane protein extraction reagent kit. The protein concentrations of cell lysates and membrane proteins were measured using a BCA protein assay kit.

Determination of $\mathrm{PIP}_{2}$ levels via dot blot analysis HepG2 cell lysates $(0.1 \mu \mathrm{g}$ of protein in $2 \mu \mathrm{l})$ were spotted onto Hybond-P polyvinylidene difluoride membranes at the center of a grid drawn by pencil. The membrane was blocked with 5\% ECL blocking reagent in Tris-buffered saline with $0.05 \%$ Tween 20 (TBS-T) for $60 \mathrm{~min}$ at room temperature. The membranes were incubated with primary antibodies for $\mathrm{PIP}_{2}(1: 10,000[v / v])$ in TBS-T for $60 \mathrm{~min}$ at room temperature with gentle shaking. The 
membrane was washed three times for 10 min each with TBS-T. The membrane was subsequently incubated with HRP-conjugated anti-mouse secondary antibody $(1: 10,000[v / v])$ in TBS-T for 60 min at room temperature with gentle shaking and then washed three times for 10 min each. The immunoreactive dots were visualized using ECL Prime western blotting detection reagent with EzCapture (ATTO, Tokyo, Japan). The densitometric intensity of the dot blot bands was calculated using CS analyzer (ATTO).

\section{Western blot analysis}

Western blotting was performed as described previously(34). Membrane proteins (5 $\square \mathrm{g}$ ) were diluted with loading buffer, denatured at $95^{\circ} \mathrm{C}$ for 3 min, and resolved using $6 \%$ sodium dodecyl sulfatepolyacrylamide gel electrophoresis (SDS-PAGE). The SDS-PAGE gel included $20 \square \mathrm{M}$ Phos-tag for recognition of activated (phosphorylated) radixin ( $p$ radixin). Proteins were transferred to a polyvinylidene fluoride membrane and subjected to semidry blotting using a Transblot SD (Bio-Rad, Hercules, CA, USA). Radixin, $p$-radixin, and $\square$ actin protein levels were detected using ECL Prime western blotting detection reagent with Ez-Capture. The densitometric intensity of each band was calculated using a CS analyzer.

\section{STATISTICAL ANALYSIS}

Data are shown as the mean \pm standard error of the mean. Significance of differences between mean values was determined by Dunnett's test after analysis of variance using GraphPad Prism software (La Jolla, CA, USA). $p<0.05$ was considered to indicate statistical significance.

\section{RESULTS}

We examined the involvement of PIP5K1A, the predominant PIP5K isoform in liver, in MRP2 activity in mouse primary hepatocytes using siRNA. To first confirm the efficiency of siPIP5K1A in depleting PIP5K1A in mouse primary hepatocytes, we evaluated the mRNA levels of PIP5K1A in cells transfected with siPIP5K1A for $48 \mathrm{~h}$. The results confirmed that PIP5K1A mRNA levels were lower after treatment with both 5 and $50 \mathrm{pmol} /$ well siPIP5K1A than the levels in control cells (Figure
1A). We selected $5 \mathrm{pmol} /$ well for use in subsequent studies since the two tested concentrations had similar depletion efficiencies.

We next evaluated MRP2 activity in mouse primary hepatocytes following PIP5K1A depletion by measuring the intracellular accumulation of $\mathrm{CDF}$ (a MRP2 substrate). An increased intracellular CDF accumulation was observed in siPIP5K1A-treated cells compared with controls (Figure 1B).

We next examined the consequence of knocking down each isoform of PIP5K on MRP2 activity in PANC1 and HepG2 cancer cell lines. We previously confirmed that PANC1 and HepG2 cells have sufficient MRP2 activity to determine the effects of PIP5K knockdown (data not shown). In PANC1 cells, the mRNA levels of PIP5K1A and PIP5K1C, but not those of PIP5K1B, were reduced by siRNA (Figure 2A). Intracellular CDF was increased in PANC1 cells transfected with siPIP5K1A and siPIP5K1C or MK571 (an MRP2 inhibitor) compared with controls (Figure 2A). In HepG2 cells, the mRNA and protein levels of PIP5K1A, PIP5K1B, and PIP5K1C were decreased by siRNA treatment compared with controls (Figure 2B). Intracellular $\mathrm{CDF}$ was also increased by siPIP5K1A and siPIP5K1C treatment in HepG2 cells.

The effects of PIP5K1 depletion on the activity of P-gp, another efflux transporter involved in multidrug resistance with chemotherapy, were also examined. PIP5K1 depletion had little effect on intracellular accumulation of R123 (a P-gp substrate) (Figure 3A) and P-gp mRNA levels (Figure 3B) in HepG2 cells.

We next examined PIP $_{2}$ amounts in HepG2 cells depleted for PIP5K1A or PIP5K1C by dot blot analysis. $\mathrm{PIP}_{2}$ levels were unchanged between control and PIP5K1A- or PIP5K1C-depleted HepG2 cells (Figure 4). To clarify whether the levels of MRP2 were affected by PIP5K1A or PIP5K1C depletion in the absence of changes of $\mathrm{PIP}_{2}$ levels, the mRNA levels of MRP2 and protein levels of radixin and $p$-radixin were determined. Neither PIP5K1A nor PIP5K1C depletion had significant effects on MRP2 mRNA levels in HepG2 cells (Figure 5A). Previous studies showed that $p$-radixin is involved in MRP2 activity(35). We found that $p$ radixin expression was decreased by PIP5K1A and PIP5K1C depletion, although the total levels of radixin were unchanged (Figure 5B). 
(A)

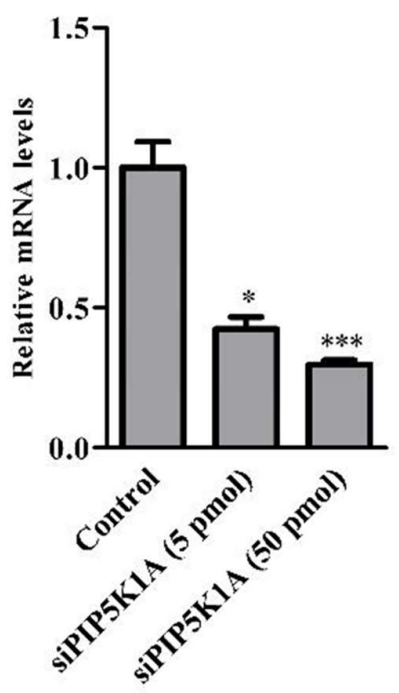

(B)

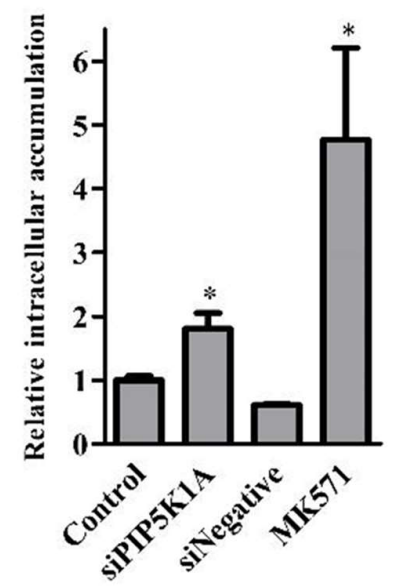

Figure 1. PIP5K1A knockdown in mouse primary hepatocytes significantly increases the intracellular accumulation of the MK571 MRP2 substrate. Relative PIP5K1A mRNA levels (A) and relative intracellular CDF accumulation (B) $48 \mathrm{~h}$ after siRNA transfection of mouse primary hepatocytes $(\mathrm{n}=3-7)$. MK571, an MRP inhibitor. ${ }^{*} p<0.05$ and $* * * p<0.001$ vs. control.

(A)
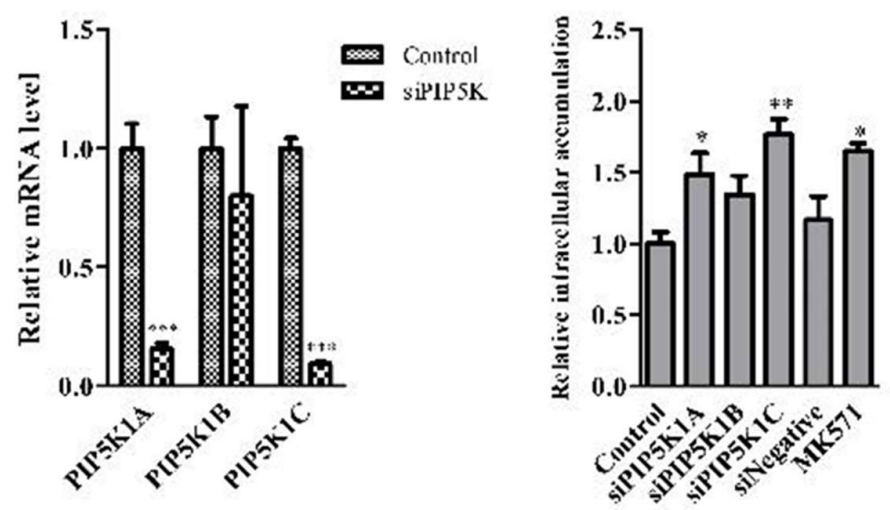

(B)
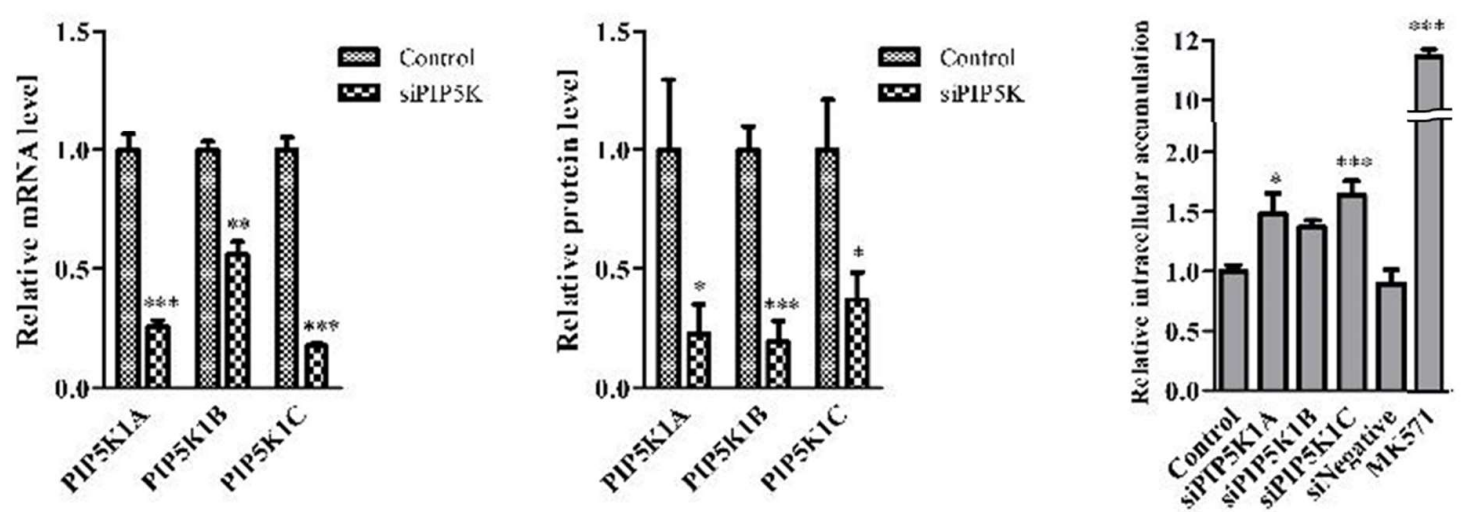

Figure 2. PIP5K1A or PIP5K1C knockdown in PANC1 and HepG2 cells significantly increases the intracellular accumulation of the MK571 MRP2 substrate. Relative mRNA and protein levels of PIP5K1A, PIP5K1B, and PIP5K1C and relative intracellular CDF accumulation in PANC1 cells (A) and HepG2 cells (B) $48 \mathrm{~h}$ after siRNA transfection ( $\mathrm{n}=$ 3-9). MK571, an MRP inhibitor. ${ }^{*} p<0.05,{ }^{* *} p<0.01$ and ${ }^{* * *} p<0.001$ vs. control. 
(A)

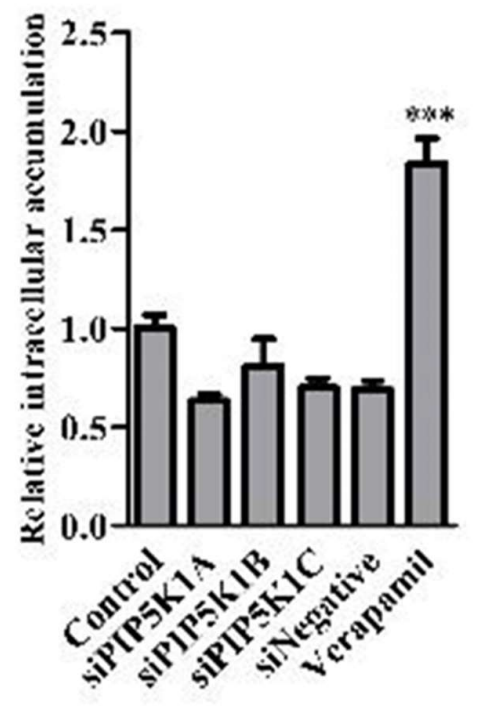

(B)

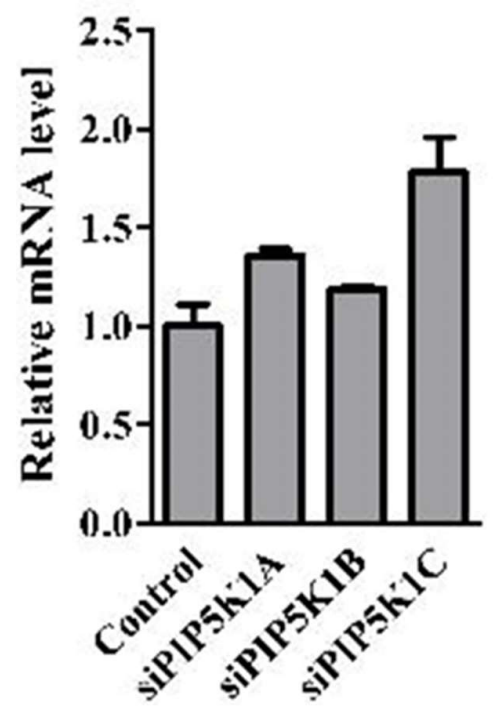

Figure 3. Intracellular accumulation of P-gp substrate and P-gp mRNA levels are unchanged by PIP5Ks knockdown. Relative intracellular R123 accumulation (A) and relative P-gp mRNA levels (B) in HepG2 cells 48 h after siRNA transfection $(\mathrm{n}=4)$. Verapamil, a P-gp inhibitor. $* * * p<0.001$ vs. control.
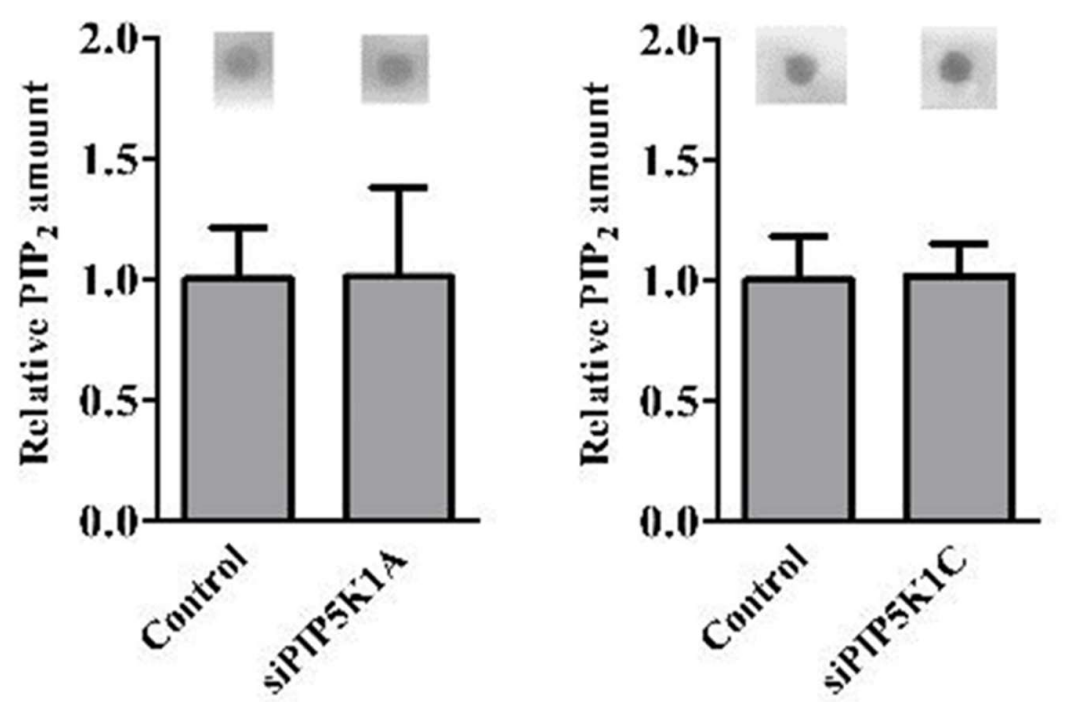

Figure 4. $\mathrm{PIP}_{2}$ amounts are unchanged by PIP5K1A or PIP5K1C knockdown. Relative $\mathrm{PIP}_{2}$ amounts in cell lysates of HepG2 cells $48 \mathrm{~h}$ after siPIP5K1A or siPIP5K1C transfection $(\mathrm{n}=3)$. 
(A)

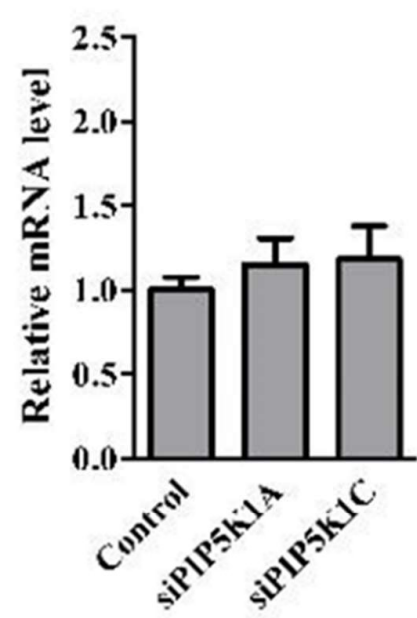

(B)

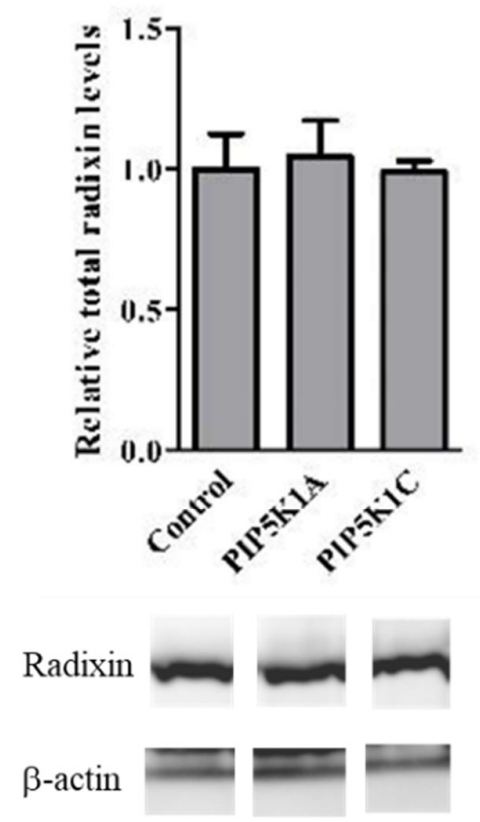

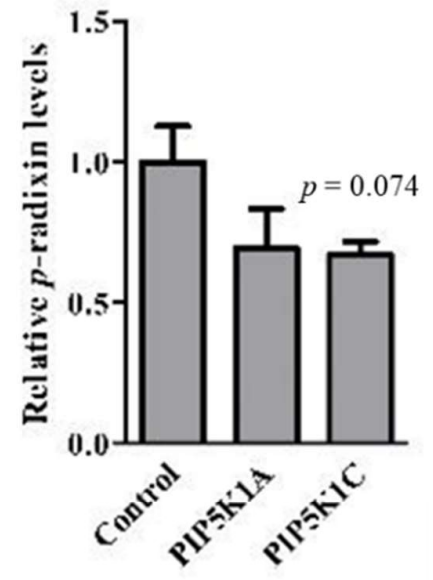

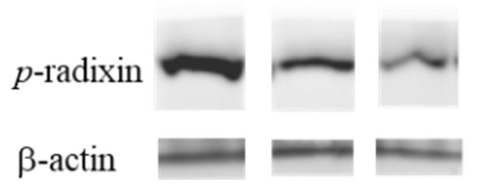

Figure 5. Levels of activated radixin tend to decrease without changes of MRP2 mRNA and radixin protein levels. Relative MRP2 mRNA levels in cell lysates (A) and relative radixin and activated radixin ( $p$-radixin) levels in membrane proteins (B) $48 \mathrm{~h}$ after siRNA transfection in HepG2 cells $(\mathrm{n}=3)$.

\section{DISCUSSION}

Inactive ERM in the cytoplasm undergoes conformational changes upon binding with $\mathrm{PIP}_{2}$, and subsequent $\mathrm{PIP}_{2}$-mediated phosphorylation converts ERM to its active form. Active ERM proteins act as scaffold proteins for MRP2. PIP5K1 is the predominant enzyme for $\mathrm{PIP}_{2}$ biosynthesis. Here we examined whether PIP5K1A, PIP5K1B, and PIP5K1C may serve as target molecules for modulating MRP2 activity in hepatocytes and cancer cells.

In the liver, PIP5K1A is the predominant enzyme for converting PI(4)P to $\mathrm{PIP}_{2}$ (24). PIP5K1A depletion resulted in approximately 2 -fold greater intracellular CDF accumulation in mouse primary hepatocytes compared with controls (Figure 1), suggesting that MRP2 activity was decreased by PIP5K1A depletion in mouse primary hepatocytes. This result suggests that this $\mathrm{PIP}_{2}$-mediated process could be a target for modulating MRP2 activity in liver.

Intracellular CDF accumulation was significantly increased by PIP5K1A or PIP5K1C depletion in HepG2 cells (Figure 2B), indicating that reducing PIP5K1A and PIP5K1C levels led to decreased MRP2 activities in HepG2 cells. These results may reflect that radixin predominantly interacts with MRP2 in cancer cells as well as in hepatocytes(36,37). However, P-gp activity in HepG2 cells as estimated by intracellular R123 accumulation was not affected by PIP5K depletion (Figure 3A). These results indicate that PIP5K1A and PIP5K1C are closely related to the transport activities of MRP2 but not P-gp. We also found that MRP2 activity was decreased by PIP5K1A and PIP5K1C depletion in PANC-1 and HepG2 cells (Figure 2A). These results suggest that PIP5K1A and PIP5K1C might participate in the modulation of MRP2 activity in cancer cells. Notably, PIP5K1B did not show similar results. The depletion efficiencies for siRNA targeting PIP5K1B were relatively low compared with those for PIP5K1A and PIP5K1C (Figure 2). Furthermore, PIP5K1B gene expression is extremely low in the liver, although PIP5K1B gene expression is detectable in some organs such as the brain and testes(38-40). Therefore, PIP5K1B may have less contribution to the modulation of MRP2 activity.

The interactions between radixin and MRP2 in HepG2 cells have been well defined. We thus examined $\mathrm{PIP}_{2}$ levels, MRP2 mRNA levels, and 
radixin and $p$-radixin levels in HepG2 cells. We also previously demonstrated that the interaction between MRP2 and radixin on the canalicular membrane in inflammation was decreased along with the ratio of $p$-radixin to total radixin(41). Our results showed that MRP2 activity in HepG2 cells was reduced by PIP5K1A and PIP5K1C depletion without alterations of $\mathrm{PIP}_{2}$ levels in the cell lysate. PIP is phosphorylated by PIP4K2 and $\mathrm{PIP}_{3}$ is dephosphorylated by PTEN in the $\mathrm{PIP}_{2}$ generation pathway(20,21). Following PIP5K1A depletion, $\mathrm{PIP}_{3}$ levels were decreased, and $\mathrm{PIP}_{2}$ levels were unchanged. A previous report showed that PIP5K1C depletion had no impact on both $\mathrm{PIP}_{3}$ and $\mathrm{PIP}_{2}$ levels(42). Our findings of unchanged $\mathrm{PIP}_{2}$ levels following PIP5K1A or PIP5K1C depletion are consistent with previous results. We also found that PIP5K1A or PIP5K1C depletion had no impact on MRP2 mRNA and radixin protein levels (Figure 5). The decreased $p$-radixin expression could be partly involved in the reduction of MRP2 activities in HepG2 cells.

A previous report demonstrated that PIP5K1A overexpression resulted in malignant progression in cancer cells(23). Our findings indicate that the knockdown of PIP5K1A or PIP5K1C could be effective in the modulation of MRP2 activity in HepG2 cells, although further studies are needed to clarify the precise mechanism. Together these results suggest that PIP5K1 depletion in cancer cells could be advantageous because the resulting effects on MRP2 activity may prevent anti-cancer drug resistance and malignant progression.

In conclusion, PIP5K1A or PIP5K1C depletion resulted in reduced MRP2 activity in hepatocytes and cancer cells without effects on $\mathrm{PIP}_{2}$ levels, suggesting that PIP5K1A and PIP5K1C could be target proteins for modulating MRP2 function.

\section{CONFLICT OF INTEREST}

The authors declare no conflict of interest.

\section{ACKNOWLEDGEMENTS}

This work was supported by JSPS KAKENHI Grant Number 18K06806, the Japanese Ministry of Education, Culture, Sports, Science, and Technology (MEXT). This research was also supported in part by the MEXT-Supported Program for the Strategic Research Foundation at Private Universities, 20142018 (S1411037). We thank Joe Barber Jr., PhD, from Edanz Group (www.edanzediting.com/ac) for editing a draft of this manuscript.

\section{REFERENCES}

1. Cole SP, Deeley RG. Multidrug resistance mediated by the ATP-binding cassette transporter protein MRP. Bioessays. 1998;20(11):931-40.

2. Bradley G, Ling V. P-glycoprotein, multidrug resistance and tumor progression. Cancer Metastasis Rev. 1994;13(2):223-33.

3. Bates SE, Robey R, Miyake K, Rao K, Ross DD, Litman T. The role of half-transporters in multidrug resistance. J Bioenerg Biomembr. 2001;33(6):50311.

4. Löscher W, Potschka H. Blood-brain barrier active efflux transporters: ATP-binding cassette gene family. NeuroRx. 2005;2(1):86-98.

5. Stieger B, Fattinger K, Madon J, Kullak-Ublick GA, Meier PJ. Drug- and estrogen-induced cholestasis through inhibition of the hepatocellular bile salt export pump (Bsep) of rat liver. Gastroenterology. 2000;118(2):422-30.

6. Gottesman MM, Fojo T, Bates SE. Multidrug resistance in cancer: role of ATP-dependent transporters. Nat Rev Cancer. 2002;2(1):48-58.

7. Fievet BT, Gautreau A, Roy C, Del Maestro L, Mangeat P, Louvard D, et al. Phosphoinositide binding and phosphorylation act sequentially in the activation mechanism of ezrin. $\mathrm{J}$ Cell Biol. 2004;164(5):653-9.

8. Ben-Aissa K, Patino-Lopez G, Belkina N V., Maniti O, Rosales T, Hao J-J, et al. Activation of Moesin, a Protein That Links Actin Cytoskeleton to the Plasma Membrane, Occurs by Phosphatidylinositol 4,5bisphosphate (PIP2) Binding Sequentially to Two Sites and Releasing an Autoinhibitory Linker. J Biol Chem. 2012;287(20):16311-23.

9. Morales FC, Takahashi Y, Momin S, Adams H, Chen X, Georgescu M-M. NHERF1/EBP50 Head-to-Tail Intramolecular Interaction Masks Association with PDZ Domain Ligands. Mol Cell Biol. 2007;27(7):2527-37.

10. Nakamura N, Oshiro N, Fukata Y, Amano M, Fukata M, Kuroda S, et al. Phosphorylation of ERM proteins at filopodia induced by $\mathrm{Cdc} 42$. Genes Cells. 2000;5(7):571-81.

11. Oshiro N, Fukata Y, Kaibuchi K. Phosphorylation of moesin by rho-associated kinase (Rho-kinase) plays a crucial role in the formation of microvilli-like structures. J Biol Chem. 1998;273(52):34663-6.

12. Pietromonaco SF, Simons PC, Altman A, Elias L. Protein kinase $\mathrm{C}$-theta phosphorylation of moesin in the actin-binding sequence. J Biol Chem. 1998;273(13):7594-603.

13. Tsukita S, Yonemura S. Cortical actin organization: lessons from ERM (ezrin/radixin/moesin) proteins. J Biol Chem. 1999;274(49):34507-10.

14. Fiévet B, Louvard D, Arpin M. ERM proteins in epithelial cell organization and functions. Biochim Biophys Acta. 2007;1773(5):653-60.

15. Suh B-C, Hille B. Regulation of ion channels by phosphatidylinositol 4,5-bisphosphate. Curr Opin 
Neurobiol. 2005;15(3):370-8.

16. Suh B-C, Hille B. PIP2 is a necessary cofactor for ion channel function: how and why? Annu Rev Biophys. 2008;37(1):175-95.

17. Kobori T, Harada S, Nakamoto K, Tokuyama S. Changes in PtdIns(4,5)P2 induced by etoposide treatment modulates small intestinal P-glycoprotein via radixin. Biol Pharm Bull. 2014;37(7):1124-31.

18. Mao YS, Yin HL. Regulation of the actin cytoskeleton by phosphatidylinositol 4-phosphate 5 kinases. Pflugers Arch. 2007;455(1):5-18.

19. Rozenvayn N, Flaumenhaft R. Phosphatidylinositol 4,5-bisphosphate mediates $\mathrm{Ca} 2+$-induced platelet alpha-granule secretion: evidence for type II phosphatidylinositol 5-phosphate 4-kinase function. J Biol Chem. 2001;276(25):22410-9.

20. Clarke JH, Irvine RF. The activity, evolution and association of phosphatidylinositol 5-phosphate 4kinases. Adv Biol Regul. 2012;52(1):40-5.

21. Campbell RB, Liu F, Ross AH. Allosteric activation of PTEN phosphatase by phosphatidylinositol 4,5bisphosphate. J Biol Chem. 2003;278(36):33617-20.

22. D'Angelo G, Vicinanza M, Di Campli A, De Matteis MA. The multiple roles of PtdIns(4)P -- not just the precursor of PtdIns(4,5)P2. J Cell Sci. 2008;121(Pt 12):1955-63.

23. Semenas J, Hedblom A, Miftakhova RR, Sarwar M, Larsson R, Shcherbina L, et al. The role of PI3K/AKT-related PIP5K $1 \alpha$ and the discovery of its selective inhibitor for treatment of advanced prostate cancer. Proc Natl Acad Sci U S A. 2014;111(35):E3689-98.

24. Xie Z, Chang SM, Pennypacker SD, Liao E-Y, Bikle DD. Phosphatidylinositol-4-phosphate 5-kinase 1alpha mediates extracellular calcium-induced keratinocyte differentiation. Mol Biol Cell. 2009;20(6):1695-704.

25. Sasaki J, Sasaki T, Yamazaki M, Matsuoka K, Taya C, Shitara H, et al. Regulation of anaphylactic responses by phosphatidylinositol phosphate kinase type I \{alpha\}. J Exp Med. 2005;201(6):859-70.

26. Nakano-Kobayashi A, Yamazaki M, Unoki T, Hongu $\mathrm{T}$, Murata $\mathrm{C}$, Taguchi R, et al. Role of activation of PIP5Kgamma661 by AP-2 complex in synaptic vesicle endocytosis. EMBO J. 2007;26(4):1105-16.

27. Song X, Yang J, Hirbawi J, Ye S, Perera HD, Goksoy E, et al. A novel membrane-dependent on/off switch mechanism of talin FERM domain at sites of cell adhesion. Cell Res. 2012;22(11):1533-45.

28. Singhal RL, Prajda N, Yeh YA, Weber G. 1Phosphatidylinositol 4-phosphate 5-kinase (EC 2.7.1.68): a proliferation- and malignancy-linked signal transduction enzyme. Cancer Res. 1994;54(21):5574-8.

29. Seglen PO. Preparation of isolated rat liver cells. Methods Cell Biol. 1976;13:29-83.

30. Kawase A, Fujii A, Negoro M, Akai R, Ishikubo M, Komura H, et al. Differences in cytochrome P450 and nuclear receptor mRNA levels in liver and small intestines between SD and DA rats. Drug Metab Pharmacokinet. 2008;23(3):196-206.

31. Kawase A, Yamada A, Gamou Y, Tahara C, Takeshita F, Murata K, et al. Increased effects of ginsenosides on the expression of cholesterol $7 \alpha-$ hydroxylase but not the bile salt export pump are involved in cholesterol metabolism. J Nat Med. 2013;67(3):545-53.

32. Kawase A, Tateishi S, Kazaoka A. Profiling of hepatic metabolizing enzymes and nuclear receptors in rats with adjuvant arthritis by targeted proteomics. Biopharm Drug Dispos. 2018;39(6):308-14.

33. Kawase A, Kaneto A, Ishibashi M, Kobayashi A, Shimada H, Iwaki M. Involvement of diclofenac acyl- $\beta$ - $d$-glucuronide in diclofenac-induced cytotoxicity in glutathione-depleted isolated murine hepatocytes co-cultured with peritoneal macrophages. Toxicol Mech Methods. 2018;1-8.

34. Kawase A, Araki Y, Ueda Y, Nakazaki S, Iwaki M. Impact of a high-cholesterol diet on expression levels of Niemann-Pick C1-like 1 and intestinal transporters in rats and mice. Eur J Drug Metab Pharmacokinet. 2016;41(4):457-63.

35. Suda J, Zhu L, Karvar S. Phosphorylation of radixin regulates cell polarity and Mrp-2 distribution in hepatocytes. Am J Physiol Cell Physiol. 2011;300(3):C416-24.

36. Kikuchi S, Hata M, Fukumoto K, Yamane Y, Matsui $\mathrm{T}$, Tamura A, et al. Radixin deficiency causes conjugated hyperbilirubinemia with loss of Mrp2 from bile canalicular membranes. Nat Genet. 2002;31(3):320-5.

37. Sekine S, Ito K, Saeki J, Horie T. Interaction of Mrp2 with radixin causes reversible canalicular Mrp2 localization induced by intracellular redox status. Biochim Biophys Acta - Mol Basis Dis. 2011;1812(11):1427-34.

38. Bayot A, Rustin P. Friedreich's Ataxia, Frataxin, PIP5K1B: Echo of a Distant Fracas. Oxid Med Cell Longev. 2013;2013:1-7.

39. Loijens JC, Anderson RA. Type I phosphatidylinositol-4-phosphate 5-kinases are distinct members of this novel lipid kinase family. J Biol Chem. 1996;271(51):32937-43.

40. Hasegawa H, Noguchi J, Yamashita M, Okada R, Sugimoto R, Furuya M, et al. Phosphatidylinositol 4phosphate 5-kinase is indispensable for mouse spermatogenesis. Biol Reprod. 2012;86(5):136, 1-12.

41. Kawase A, Sakata M, Yada N, Nakasaka M, Shimizu $\mathrm{T}$, Kato $\mathrm{Y}$, et al. Decreased Radixin Function for ATP-Binding Cassette Transporters in Liver in Adjuvant-Induced Arthritis Rats. J Pharm Sci. 2014;103(12):4058-65.

42. Choi S, Hedman AC, Sayedyahossein S, Thapa N, Sacks DB, Anderson RA. Agonist-stimulated phosphatidylinositol-3,4,5-trisphosphate generation by scaffolded phosphoinositide kinases. Nat Cell Biol. 2016;18(12):1324-35. 